
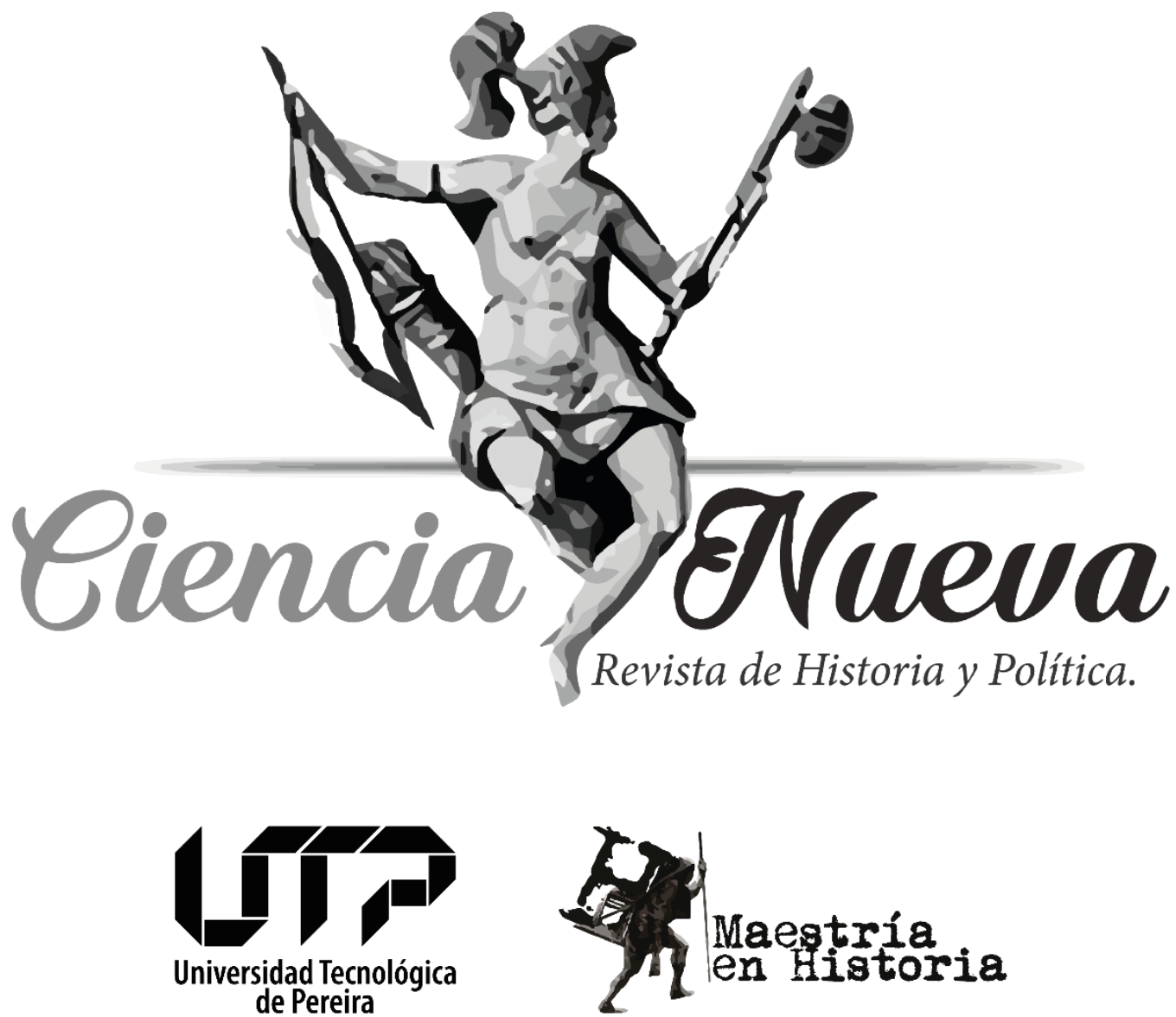

RESEÑAS

BETANCOURT, ALEXANDER. AMÉRICA LATINA: CULTURA LETRADA Y ESCRITURA DE LA HISTORIA. BARCELONA: ANTHROPOS EDITORIAL, UNIVERSIDAD AUTÓNOMA DE SAN LUIS POTOSÍ, MÉXICO, 2018 BETANCOURT, ALEXANDER. LATIN AMERICA: LITERATE CULTURE AND HISTORY WRITING. BARCELONA: ANTHROPOS EDITORIAL, UNIVERSIDAD AUTÓNOMA DE SAN LUIS POTOSÍ, MÉXICO, 2018

Jhoan Manuel Camargo Suaza pp. $104-108$

Vol. 4 Núm. 1 | Enero-Junio de 2020 Pereira, Colombia 


\title{
BETANCOURT, ALEXANDER. AMÉRICA LATINA: CULTURA LETRADA Y ESCRITURA DE LA HISTORIA. BARCELONA: ANTHROPOS EDITORIAL, UNIVERSIDAD AUTÓNOMA DE SAN LuIS Potosí, MÉXICO, 2018*
}

\author{
BETANCOURT, ALEXANDER. LATIN AMERICA: LITERATE CULTURE AND HISTORY WRITING. BARCELONA: \\ Anthropos Editorial, Universidad Autónoma de SAn Luis Potosí, México, 2018
}

Jhoan Manuel Camargo Suaza**

jhoancamargo1984@gmail.com

ORCID: http://orcid.org/0000-0002-8134-5873

\begin{aligned} & \hline Recibido: 24 de noviembre de 2019. \\ & Revisado: 20 de febrero de 2020. \\ & Aceptado: 28 de mayo de 2020. \\ & Publicado: 04 de septiembre de 2020. \\ & \hline\end{aligned}

U no de los aportes más relevantes de la historiografía contemporánea, sin lugar a dudas, redunda en el entendimiento de la conformación de los Estados nacionales latinoamericanos. Cómo funciona el Estado y qué lo diferencia de las experiencias de otras latitudes ha sido una incertidumbre que ha movido a historiadores connacionales a desarrollar toda suerte de estrategias, así como abordar otras tantas perspectivas en pos de dar explicaciones acuciosas en estas lindes.

En esta perspectiva surgen propuestas como la realizada por Alexander Betancourt Mendieta, cuya labor a cerca de la naturaleza y conformación del Estado latinoamericano se ha ido construyendo ya desde experiencias anteriores como en el caso de Historia y nación $(2007)^{306}$. Asimismo, otros trabajos posteriores han estado encaminados no solo a identificar el papel de las instituciones, así como las coyunturas históricas en el proceso de conformación del Estado y la nación, sino que, más allá de eso, han aportado para entender el papel de las comunidades letradas y otros estamentos incipientes de orden académico, y si se quiere científico, en dicha empresa.

En ese sentido, es menester reconocer que por su trabajo, Betancourt Mendieta logra establecer una imagen del Estado más orgánica y alejada de máculas sobre prohombres, estructuras deshumanizadas o procesos amañados por parte de ciertos discursos políticos al momento de explicar su conformación; es decir, trasciende esa mirada estática y simplista para mostrar un Estado en permanente construcción, conformado robustamente por diferentes actores, instituciones y procesos que lo hacen un elemento mutable y sobre todo complejo.

En esta ocasión, ha correspondido abordar el libro América Latina: cultura letrada y escritura de la historia, obra publicada en 2018 por Anthropos Editorial con el apoyo del

\footnotetext{
* Este documento respeta las directrices y normas dispuestas en la Declaración de Ética de Publicación de Ciencia Nueva, Revista de Historia y Política. Esta declaración puede consultarse en la página web de la revista: http://revistas.utp.edu.co/index.php/historia

${ }^{* *}$ Licenciado en Historia de la Universidad del Valle, y candidato a Magíster en Historia de la Universidad Tecnológica de Pereira.

${ }^{306}$ Alexander Betancourt, Historia y nación. Tentativas de la escritura de la historia en Colombia (Medellín: La Carreta Editores E.U., Universidad Autónoma de San Luis Potosí, Coordinación de Ciencias Sociales y Humanidades de la UNAM, 2007).
} 
Departamento de Ciencias Sociales y Humanidades de la Universidad Autónoma de San Luis Potosí de la cual Alexander Betancourt hace parte como docente.

Desde la introducción, el libro se plantea ambicioso, toda vez que los objetivos manifiestos por el autor se difunden en una introducción amena y bien lograda. Allí confluyen diferentes temas historiográficos, pero también teóricos, a saber: el papel de la escritura, los hombres de letras, la conformación de los Estados nacionales entre finales del siglo XIX y principios del XX. Así como la revisión de los aportes de historiadores como Langlois, Seignobos o Ranke, ya no desde la rigidez positivista, sino desde una postura más reflexiva y sobre todo útil, según argumentos del propio autor:

De hecho, al aproximarse a los trabajos denostados como «metodistas» puede encontrarse que merecen mayor atención [...] el trabajo realizado por aquellos «metódicos» fue una contribución de primer orden y, por lo tanto, no podemos incurrir en su condena o en la alusión desinformada ${ }^{307}$.

En cuanto al papel de la escritura, el autor plantea lo siguiente:

Es importante analizar cómo la escritura como actividad intelectual, al carecer de espacios institucionales específicos para su desenvolvimiento especializado, entra a formar parte de la cultura letrada que no se caracteriza por la precisión de sus límites y campos de acción, y confluyen en las tareas asumidas por hombres de letras. Por esta razón, no es extraño que en instituciones incipientes como las academias de Historia y sus miembros recayeran por igual, y sin problematizarse, tareas que hoy se entenderían como etnológicas, museográficas, archivísticas, restauracionistas, editoriales, además de las que involucraban la escritura sobre el pasado ${ }^{308}$.

Más allá de los juegos de citaciones, se puede decir que el objeto principal de estudio de este libro es el papel de la escritura y los hombres de letras a finales del siglo XIX y principios del XX y su injerencia o, en otras palabras, el usufructo del Estado de dicha actividad y sujetos. Sin embargo, en honor a la verdad, los alcances y objetivos van más allá de ello en la medida en que se puede observar cómo hay un interés no solo en la correlación entre la escritura, los hombres de letras y el Estado, sino que Betancourt de igual manera involucra otras inquietudes como el papel de la escritura en la construcción del relato nacional, la disposiciones administrativas del Estado en relación con las instituciones académicas y de pensamiento, pero también la situación en diferentes espacios geográficos en el mismo periodo.

Por lo anterior, no es un libro cuyas tesis reposan en una línea argumental tranquila, sino que se bifurca en las cuitas investigativas del autor, sin decir con ello que es un trabajo desordenado o poco profundo. Lo que se pretende señalar es que, si bien hay una preocupación por demostrar el acento en el papel de la cultura letrada y la escritura de la historia, como tiene a bien el título, hay marcados espacios de disertación en relación con el Estado, la academia, la nación y los discursos nacionales. En ese orden de ideas, el documento adquiere un cariz polifónico que se hila con acierto, así por momentos intensifique el análisis en otros linderos investigativos.

No obstante, la crítica anterior, el cúmulo de lecturas, autores y referencias es lo suficientemente holgado para que dicha apuesta no preocupe y, por el contrario, invite al lector a debatir o enriquecer sus propias preocupaciones con otros campos de interés del autor

307 Alexander Betantourt, América latina: cultura letrada y escritura de la historia (Barcelona: Anthropos Editorial, Universidad Autónoma de San Luis Potosí, México, 2018), 10.

${ }^{308}$ Betantourt, América latina..., 13. 
y a los cuales ya tiene acostumbrados a lectores de obras anteriores. No en balde el texto se divide en cinco capítulos breves, que no palidecen en cuanto a estructura y aporte; por tal motivo, vale la pena detenerse a esbozar la naturaleza o contenido de dichos capítulos.

Cap. I. El pasado y la escritura. El autor plantea la necesidad de los núbiles estados modernos, cuyos preceptos ideológicos buscan separarse del pasado colonial. En ese sentido, la idea del ciudadano, la patria y la unidad de una población bajo unas delimitaciones y territorios soberanos se busca introducir y propagar a partir de los hombres de letras, de ahí la importancia de estos y sus discursos con relación al establecimiento de la idea de Estado, nación, patria, etc. En dicho afán, Betancourt argumenta que los esfuerzos, tanto del Estado como de los letrados, se encaminaron en temáticas específicas como la política, la historia y la guerra, dejando de lado la posibilidad de introducirse en temáticas relacionadas con el conocimiento científico.

Cap. II. La escritura de la historia: el pasado, las instituciones y la ciencia. Este apartado hace hincapié en las sociedades letradas de finales del siglo XIX y su contribución en la construcción del Estado nacional americano, en tanto que sus divagaciones y estudios, las más de las veces autónomos, fueron usufructuados y en algunos casos financiados por los nacientes Estados, aclarando que dichos colectivos eran de carácter privado. Inquietudes sobre botánica, cartografía, fisiología o literatura, más allá de constituirse como aportes científicos o humanísticos eran pensados como aportes producto del deber hacia la patria por parte de estos hombres, quienes se pensaban a sí mismos como los ciudadanos de la República de las letras y no abanderados de la construcción de la cultura nacional. A lo largo de este capítulo se explica el tránsito paulatino de dichas sociedades a instituciones y colegios más formalizados y administrados por el Estado.

Cap. III. Del momento letrado: hombres de letras y revistas. Si el capítulo anterior habla sobre la transición de las sociedades letradas y el surgimiento de espacios de pensamiento más académicos e institucionales, este se concentra en un principio en el cambio de la escritura para principios de siglo XX; es decir, cómo el ejercicio de escribir muta debido a sus funciones y temáticas. En ese sentido, los formatos de escritura, la figura del autor, así como la creación y la ampliación del público letrado redunda en la creación de revistas y periódicos, y con ello una suerte de revolución en las formas de comunicar. No deja de ser interesante cómo se describe el papel del hombre de letras y cómo su actividad fluctúa entre la literatura, la política y el mundo del conocimiento en general. Lo anterior, debido a la ausencia de espacios de especialización y la posibilidad de estos hombres de letras de poder hablar de todo.

Cap. IV. De las revistas a las instituciones: los inicios del momento profesional. Los inicios de este capítulo se dedican a explicar el panorama internacional entre finales del siglo XIX y principios del XX (el periodo de estudio del libro) y cómo la revolución rusa, la Gran Guerra y otros tantos hechos determinan hasta cierto punto el papel que asumieron los Estados latinoamericanos en relación con Norteamérica y Europa. Posteriormente, a través de la figura de Germán Arciniegas, Betancourt retrata el proceso de transformación de la historia, su finalidad, estructura, regulación e institucionalización. De manera que los objetitos de la historia, su función y carácter científico se acercan más a la realidad actual de historia-ciencia. Entre otras intenciones, el autor hace un bellísimo intento por explicar la transformación de la historia y otras ciencias sociales a lo largo del siglo pasado.

Cap. v. La idea de América: un instituto, un proyecto y la mirada continental. Como complemento final, tanto del capítulo anterior como del libro, el autor en «La idea de América» cierra el recorrido tanto de Europa y Norteamérica como de las ciencias sociales y 
sus recientes transformaciones. A la vez, traslada sus preocupaciones de nuevo a Latinoamérica, especialmente con El Instituto Panamericano de Geografía e Historia, el cual surge como una iniciativa latinoamericana para pensar el territorio, pero también para entenderlo; los insumos técnicos y científicos de países como México se avocan a comprender el territorio y el pasado, en contraposición a las iniciativas similares en ciencias sociales y humanidades que se estaban realizando en Europa desde años anteriores. En ese sentido, esta iniciativa redunda no solo en preocupaciones científicas, sino políticas, como intento de que lo latinoamericano sobresalga en el panorama internacional.

En suma, América Latina: cultura letrada y escritura de la historia es un documento que, sobre todo, vive dos momentos. Uno que se desenvuelve en los primeros tres capítulos que relaciona la escritura, la sociedad letrada y la historia con la construcción de los Estados nacionales, a partir de allí retrata con gran habilidad el trasegar de la actividad escrita y sus actores desde las sociedades de letras hasta las instituciones, academias y universidades. A la par que mutan las formas de escritura y se especializa, desde el letrado hasta el académico, desde el literato-humanista-político, pero también científico social, hasta el periodista o el académico o el estadista. La especialización en una sola actividad conlleva a la formalización de la generación de conocimiento y reflexión.

Por otro lado, al final del libro, se puede ver un ejercicio de síntesis de gran valor en el que se expone el proceso de construcción de las ciencias sociales a través de sus debates, escuelas y coyunturas históricas. Presenta la posibilidad de poner en entredicho o reflexionar sobre ciertas posturas que ya se entendían como certezas y que Betancourt logra cuestionar con método y fuentes. Asimismo, juega con una espacialidad mucho más grande en el sentido de que logra poner en conversación a Europa y Latinoamérica, como fue uno de sus objetivos en el principio del documento. 


\section{Referencias}

Betancourt, Alexander. Historia y nación. Tentativas de la escritura de la historia en Colombia. Medellín: La Carreta Editores E.U., Universidad Autónoma de San Luis Potosí, Coordinación de Ciencias Sociales y Humanidades de la UNAM, 2007.

. América latina: cultura letrada y escritura de la historia. Barcelona: Anthropos Editorial, Universidad Autónoma de San Luis Potosí, México, 2018. 